\title{
VARIACIÓN INTERANUAL DE LA DIETA DE LA MERLUZA Merluccius gayi peruanus (GUITCHENOT) EN LA COSTA PERUANA
}

\section{INTERANNUAL VARIATION IN THE DIET OF HAKE Merluccius gayi peruanus (GUITCHENOT) ON THE PERUVIAN COAST}

\author{
Henry Orrego ${ }^{1}$ y Jaime Mendo ${ }^{2}$
}

\begin{abstract}
Resumen
Merluccius gayi peruanus es la especie demersal más importante de la pesquería industrial de la costa norte del Perú y juega un rol ecológico importante en el ecosistema. Con la finalidad de conocer el comportamiento dietario de esta especie se analizaron los contenidos estomacales de 2 836 individuos capturados a bordo de embarcaciones arrastreras industriales en la zona norte del Perú durante 1995 a 1997. La longitud total de la merluza fluctuó entre 24 y $73 \mathrm{~cm}$ y mayormente en un rango de 34 y $46 \mathrm{~cm}$. El porcentaje de estómagos con alimento en las diferentes clases de longitud fluctuaron entre 39,5 y $100 \%$. Los peces teleósteos contribuyeron con el $87 \%$ de la masa de alimento ingerido, $10.4 \%$ fueron moluscos cefalópodos y sólo el $2.6 \%$ fueron crustáceos. Entre los peces ingeridos, la misma merluza es el principal ítem alimenticio (40.6\%) evidenciando un alto grado de canibalismo, siguiéndole en importancia la familia Engraulidae (24.1\%) donde predomina claramente la anchoveta. Para el año 1997 se produce una modificación en la dieta ampliándose el espectro de la estructura alimenticia de la merluza. Basados en el IRI los grupos predominantes en la dieta correspondieron a la familia Eufausidae, la especies Engraulis ringens, Merluccius gayi y P. stephanophrys. La Plenitud total media (TFI) presenta una tendencia ascendente principalmente hasta fines de 1996, pero a inicios del año 1997 manifiesta un franco descenso. Para los años 1995 y 1996 el análisis cluster organizó a las clases de longitud de la merluza en 3 grupos compuesto por ejemplares de $30-34 \mathrm{~cm}, 34-50 \mathrm{~cm}$ y un último con merluzas grandes de 50-66 cm. Para 1997, se observan solo 2 grupos principales compuestos básicamente por merluzas de 30-46 cm y 50-66 cm, resultado que demuestra el efecto del fenómeno El Niño en el comportamiento alimentario de la merluza y su adaptación a los cambios en la oferta alimenticia.
\end{abstract}

Palabras clave Merluccius gayi peruanus, dieta alimenticia, El Niño, Perú.

\begin{abstract}
Merluccius gayi is the most important demersal species exploited by the fishery industry in the northern coast of Peru and plays an important ecological function in the ecosystem. In order to know its predatory behavior stomach content of 2836 individuals were analyzed. The individuals were caught on board of industrial trawlers in the north of Peru during 1995 to 1997 . The total lengths ranged between 24 and $73 \mathrm{~cm}$ and mainly in a range of 34 and $46 \mathrm{~cm}$. The percentage of stomachs with food in different length classes ranged between 39.5 and $100 \%$. Fishes were the largest item in the diet with $87 \%$ followed by the mollusks cephalopods with $10.4 \%$ and crustaceans with $2.6 \%$. The euphausiids were the most abundant crustacean and among the fishes ingested the hake was the main food item $(40.6 \%)$ showing a high degree of cannibalism, followed by the Engraulidae (24.1\%) clearly dominated by the anchovy. For 1997 the diet showed a wider spectrum. Based on IRI values, the predominant groups in the diet corresponded to Eufausidae, the species Engraulis ringens, Merluccius gayi and P. stephanophrys. According to the total fullness (TFI) showed a positive trend mainly until the end of 1996, however after started to decline. The cluster analysis applied to the stomachs contents sampled in 1995 and 1996 by length classes organized the individuals in 3 groups: of $30-34 \mathrm{~cm}, 34-50 \mathrm{~cm}$ and $50-66 \mathrm{~cm}$. For 1997, the cluster analysis grouped the stomachs of hake into two groups of 30-46 cm and 50-66 cm, which demonstrates the effect of El Niño on the foraging behavior and the adaptation of hake to changes in the food supply.
\end{abstract}

Key words: Merluccius gayi peruanus, feeding habits, El Niño, Peru. 


\section{Introducción.}

Merluccius gayi peruanus es un pez demersal que se distribuye frente a la costa peruana en toda la plataforma continental principalmente entre profundidades someras y hasta aquellas superiores a los 350 m (Castillo et al., 1995). Esta especie tiene una distribución latitudinal de acuerdo a su tamaño, donde los especímenes pequeños están en la parte sur y los más grandes en la parte norte. La distribución de la merluza lejos de la costa es más o menos constante y ampliamente limitada a la extensión este-oeste de la plataforma continental, mientras que la distribución norte-sur varía notablemente. Esta distribución ha sido explicada en función al contenido de oxígeno de las capas de aguas profundas, el cual se ve incrementado cuando las aguas cálidas ricas en oxígeno invaden desde el norte el ecosistema de afloramiento peruano, p.e. durante los eventos de El Niño (Muck, 1989). Esta especie representa alrededor del $80 \%$ de los desembarques de peces demersales en la costa peruana y por lo tanto juega un rol importante en el funcionamiento del ecosistema marino demersal en el norte del Perú.

La literatura disponible sobre la merluza en el Perú indica que la mayor parte de los estudios se han centrado mayormente en la evaluación de su stock, algunos aspectos biológicos y su relación con las condiciones oceanográficas (Espino, 1990; Castillo et al., 1996; Tam et al., 2006; Guevara \& WosnitzaMendo, 2009). Dentro de los aspectos biológicos varios autores han estudiado los patrones dietarios y el comportamiento alimenticio de la merluza como Castillo et al. (1989) quienes concluyen que la merluza muestra una gran preferencia por la presa sardina para individuos mayores a $20 \mathrm{~cm}$ de longitud. Así mismo, Muck (1989) sobre el consumo de anchoveta por la merluza plantea un modelo en función al tamaño de la merluza. Otros estudios se han efectuado en relación a la tasa de consumo (Muck et al., 1988; Castillo et al., 1995) como también en relación a la composición de la dieta (Alamo y Blaskovic, 1994; Alamo y Espinoza, 1996, 1997; Espinoza 2000, 2001).

A pesar de la considerable atención enfocada, relativamente poco es conocido acerca de las variaciones ontogenéticas y temporales, así como del efecto del fenómeno El Niño en el comportamiento alimenticio de esta especie. El presente estudio está dirigido a determinar el alimento y los patrones de alimentación de la merluza peruana en la costa norte del Perú y analizar las variaciones interanuales durante años normales 1995-1996 y durante el comienzo de un año considerado con presencia del evento de El Niño 1997. En este contexto se evalúa la hipótesis de una ampliación del espectro trófico de la merluza durante un evento cálido.

\section{Materiales y métodos.}

Se recolectaron un total de 2836 ejemplares de merluza peruana a bordo de las embarcaciones comerciales de la Empresa Santa Mónica equipadas con red de arrastre de fondo entre mayo de 1995 y diciembre de 1997. Las muestras fueron colectadas en estaciones de arrastre de las embarcaciones en la región norte del Perú frente al Puerto de Paita $\left(05^{\circ}\right.$ 04'S; 81 ${ }^{\circ} 06^{\prime} \mathrm{W}$ ) (Figura 1).

En todos los muestreos a cada ejemplar se le midió la longitud total LT $(\mathrm{cm})$, luego se tomó una muestra estratificada por tallas a fin de asegurar la presencia de ejemplares de todas las tallas en las muestras obtenidas. Para cada individuo se registró el peso, sexo, estadio sexual y se le extrajo el estómago los cuales fueron preservados en formalina al $5 \%$ para su posterior examen en el laboratorio.

En el laboratorio el contenido estomacal de cada individuo fue analizado y se contabilizaron los estómagos vacíos y con contenido estomacal. En el caso de los estómagos con alimento, éstos fueron limpiados, y secados con papel absorbente para luego registrar el peso y el número de presas. Las presas fueron identificadas sobre la base de la literatura de Del Solar \& Alamo (1970) para los crustáceos y Chirichigno (1998) para el caso de los peces, todos ellos hasta el menor nivel taxonómico.

La Tabla 1 muestra el número de estómagos colectados y analizados por estación del año a bordo de las embarcaciones comerciales durante 1995 y 1997.

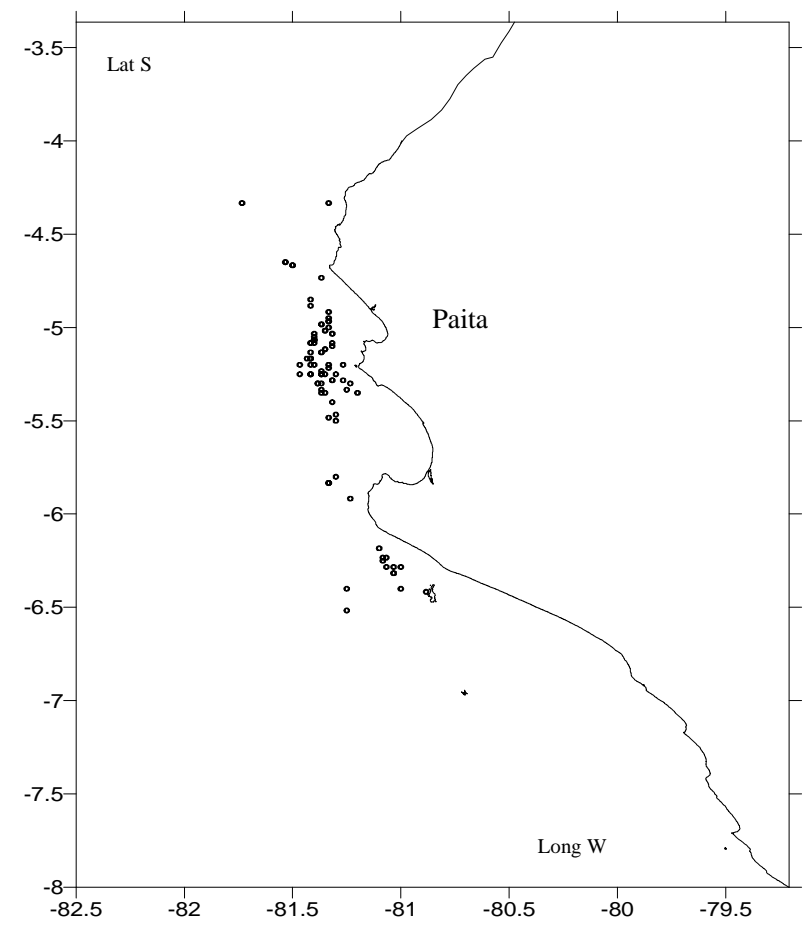

Figura 1. Estaciones de pesca comercial donde se colectaron las muestras de merluza (Merluccius gayi) durante 1995 - 1997 en el norte del Perú. 
Tabla 1. Número total de estómagos (n) por estación de los años colectados frente a Paita durante 1995 y 1997.

\begin{tabular}{l|c|c|c|c|c|c}
\hline \multirow{2}{*}{$\begin{array}{l}\text { Estación } \\
\text { del año }\end{array}$} & $\mathrm{n}$ & $\begin{array}{c}1995 \\
\text { Profundidad } \\
(\mathrm{m})\end{array}$ & $\mathrm{n}$ & $\begin{array}{c}\text { Profundidad } \\
(\mathrm{m})\end{array}$ & $\mathrm{n}$ & $\begin{array}{c}\text { Profundidad } \\
(\mathrm{m})\end{array}$ \\
\cline { 2 - 7 } & & & 418 & 152 & 274 & 202 \\
Verano & 90 & 183 & 381 & 182 & 282 & 247 \\
Otoño & 292 & 182 & 328 & 225 & 273 & 268 \\
Invierno & 153 & 150 & 290 & 178 & 55 & 253 \\
Primavera & 153 & 1417 & & 884 \\
\hline TOTAL & 535 &
\end{tabular}

Análisis y procesamiento de datos.

Se realizaron análisis de los estómagos vacíos, estómagos con alimento y evertidos, tanto en términos porcentuales por grupos de tallas en cada año de estudio, como también su comportamiento estacional por temporadas. Los datos de peso y número de las presas fueron agrupados y analizados de acuerdo a la talla de la merluza y a la estación del año. A fin de investigar los cambios temporales de la dieta los datos fueron agrupados por estaciones y con la finalidad de detectar diferencias ontogenéticas en la dieta relacionados con el tamaño de los peces, los datos del contenido estomacal fueron agrupados en clases de longitud de $4 \mathrm{~cm}$ de la merluza.

Para el periodo total de estudio, de los estómagos con alimento reconocible se estimó el porcentaje de la masa $(\% M)$ de cada una de las presas consumidas, expresada como la masa (g) de cada presa en relación a la masa total de todas las presas en el estómago; de acuerdo a esto para el análisis de la composición de las dietas, los taxa de las principales presas fueron agrupados en categorías de acuerdo a su importancia (Pillar \& Wilkinson, 1995; Silva, 1999). Igualmente se analizó estacionalmente los pesos promedios de los contenidos estomacales por grupos de longitud de la merluza. Los cambios en las dietas anuales por grupos de longitud de la merluza fueron también analizados, donde los principales grupos alimenticios estuvieron expresados como un porcentaje del total de las presas en cada grupo de longitud.

Con la finalidad de conocer la capacidad de predación de la especie merluza, se analizó la relación entre la longitud del cuerpo del predador y el peso de las presas en sus estómagos, ajustándose al mejor modelo matemático.

Para conocer la contribución de cada categoría de la presa a la dieta de la merluza en forma estacional se determinaron cuatro medidas relativas de las cantidades de presas:

Índice de Importancia Relativa (IRI) (Pinkas et al., 1971):

$$
\mathrm{IRI}=(\mathrm{Cn}+\mathrm{Cw}) \mathrm{F}
$$

donde:

F: porcentaje de frecuencia de ocurrencia

$\mathrm{Cn}$ : porcentaje de composición numérica

$\mathrm{Cw}$ : porcentaje de composición gravimétrica
Para calcular la jerarquía de las presas en la dieta se aplicó al valor de IRI el índice de jerarquización (DJ) que toma el valor más alto del IRI y calcula el porcentaje de todos los demás valores a partir de él. Si el porcentaje del ítem presa se encuentra incluido entre el $100 \%$ y $75 \%$ se le considera fundamental; si se ubica entre el $75 \%$ y $50 \%$ se le considera secundario, si está entre el $50 \%$ y $25 \%$ es accesorio, y si está por debajo del $25 \%$ se le considera accidental (Martori, 1991).

Índice de Plenitud Total media (TFI) (Paz et al., 1993):

$$
\mathrm{TFI}=1 / \mathrm{n} \sum\left(\mathrm{fw} /\left(\mathrm{f}_{\mathrm{l}}\right)^{3}\right) \times 10^{4} \quad(\text { de } 1 \text { a } \mathrm{n})
$$

donde:

n: número de estómagos examinados

fw : peso del contenido estomacal de cada pez $\mathrm{f}$ (en $\mathrm{g}$ )

$\mathrm{f}_{\mathrm{l}}$ : longitud total del pez $\mathrm{f}(\mathrm{en} \mathrm{cm})$

Índice de Plenitud Parcial media de la presa (PFI) (Paz et al., 1993):

$$
\left.\mathrm{PFI}=1 / \mathrm{n} \Sigma\left(\mathrm{fp} /\left(\mathrm{f}_{\mathrm{l}}\right)^{3}\right) \times 10^{4} \text { (de } 1 \text { a n }\right)
$$

donde:

fp : peso de la presa p en el estómago del pez f (en g)

Índice de Intensidad de Alimentación (FI):

$$
\mathrm{FI}=\mathrm{SNf} / \mathrm{SNt}
$$

donde:

SNf: número de estómagos conteniendo algún alimento

SNt: número total de estómagos analizados

Con la finalidad de identificar patrones en los datos de contenido estomacal se usaron técnicas multivariadas no paramétricas. Para ello se construyeron matrices de similaridad usando el coeficiente Bray-Curtis (Bray \& Curtis, 1957) a datos pareados aplicado a la raíz cuadrada de los datos de pesos de presas. Los pesos de presas fueron usados porque esta medida refleja mejor la importancia energética de la presa en la dieta que la frecuencia de ocurrencia o abundancia numérica. El análisis cluster fue aplicado a los datos de presas agrupados por años y por grupos de talla del predador con intervalos de 4 $\mathrm{cm}$ y por año usando el algoritmo UPGMA (unweigthed pairgroup with arithmetic mean). Para 
corroborar los resultados del cluster se aplicó la técnica multivariada de ordenación NMDS non-metric multidimensional scaling (Clarke, 1993). Todos los análisis multivariados fueron realizados con el programa Plymouth Routines in Multivariate Ecological Research (PRIMER, versión 6.0; PRIMER-E Ltd, Plymouth, UK).

\section{Resultados y discusión.}

Durante el periodo de estudio 19951997 se midieron 2836 ejemplares de merluza cuyas longitudes totales fluctuaron entre 24 y $73 \mathrm{~cm}$ y cuyo principal rango estuvo entre 34 y $46 \mathrm{~cm}$ (Figura 2). Realizando un análisis general, es notorio observar la presencia de peces con un amplio rango de tamaños para todo el periodo de estudio pero si con un notorio menor número de ejemplares para individuos menores a 30 $\mathrm{cm}$ y mayores a $58 \mathrm{~cm}$. En este sentido se asemeja a lo encontrado por Guevara (1997) que halló escasa presencia de

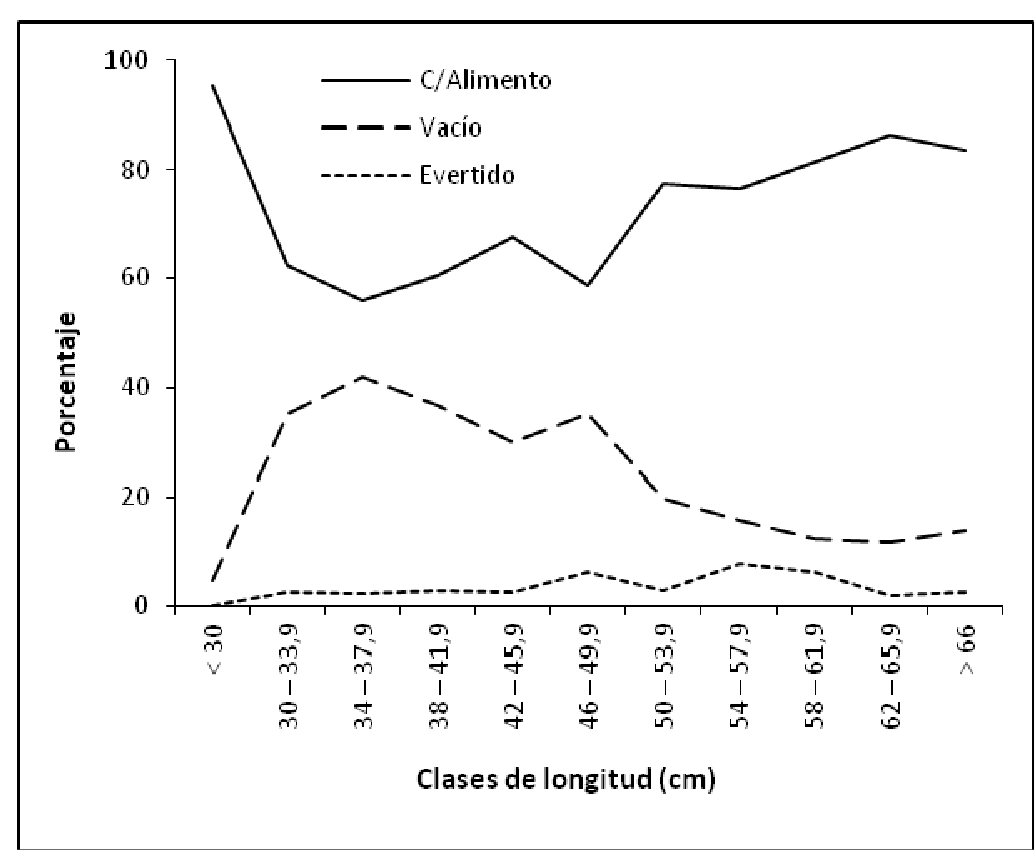

Figura 3. Porcentaje promedio de la condición de los estómagos analizados por clases de longitud durante 1995-1997. ejemplares en un rango de tamaños entre 25 y $30 \mathrm{~cm}$ ( 2 años) pero difiere en lo anotado de que existe una fuerte presencia de un grupo de juveniles con moda de 20 a $21 \mathrm{~cm}$.

La población relativamente joven por debajo de la talla de $34 \mathrm{~cm}$ concentra sólo el $16 \%$ de la población, pero es predominante los ejemplares comprendidos entre los 34 y $46 \mathrm{~cm}$ con aproximadamente el $73 \%$ de la población muestreada a diferencia de lo encontrado por Guevara \& Wosnitza (1997) que hallaron hasta un $80 \%$ de ejemplares por debajo de esta talla mínima de captura. Por otro lado, para el periodo total de estudio, se determinó que cerca del $64 \%$ de la totalidad de

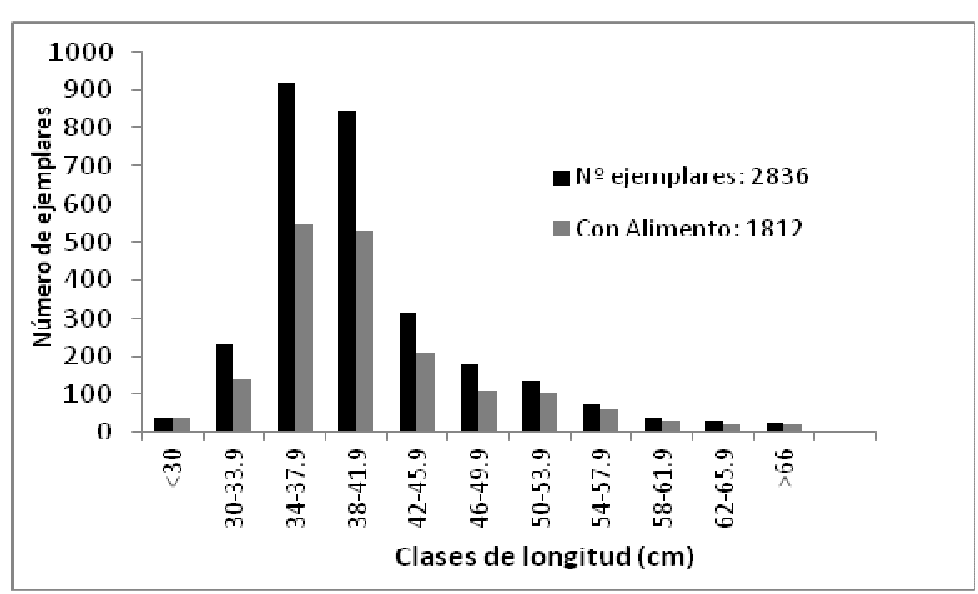

Figura 2. Número total de individuos colectados y número de individuos con estómagos con alimento por clases de longitud de M. gayi analizados en el presente estudio. individuos muestreados presentaron estómagos con contenido alimenticio.

Intensidad de alimentación.

La Figura 3 presenta para el periodo total el comportamiento del promedio de porcentajes de estómagos en cada una de las condiciones con alimento, vacíos y evertidos.

El porcentaje de estómagos con alimento fue bastante consistente para los tres años de estudio en las diferentes clases de longitud fluctuando entre 39,5 y $100 \%$. Del total de 2836 estómagos analizados 932 estómagos (32,9\%) estuvieron vacíos, 1812 estómagos $(63,9 \%)$ estuvieron con alimento y sólo $92(3,2 \%)$ se encontraron evertidos. Contrariamente, el número de estómagos evertidos fue bajo para todo el periodo de estudio oscilando entre 0 y $20 \%$, siendo notoriamente sus valores más bajos en el año1997. Para los estómagos vacíos los porcentajes muestran una tendencia decreciente cuando se examinan ejemplares grandes alcanzando sus valores mínimos entre las tallas 58,0 y $65,9 \mathrm{~cm}$.

Del mismo modo, analizando las tendencias estacionales de la plenitud de los estómagos de la merluza durante el periodo de estudio (Figura 4), se muestra una tendencia decreciente de los estómagos vacíos en contraste con los estómagos que contienen alimento que denotan una tendencia creciente, demostrando de esta manera al final del 
periodo una mayor oferta alimenticia en la zona de captura de esta especie, sobre todo en los meses de invierno y primavera del año 1997 que se caracterizó por ser un año en que se presentaron las anomalías de un evento como El Niño. Los estómagos evertidos tienen un comportamiento irregular durante los tres años.

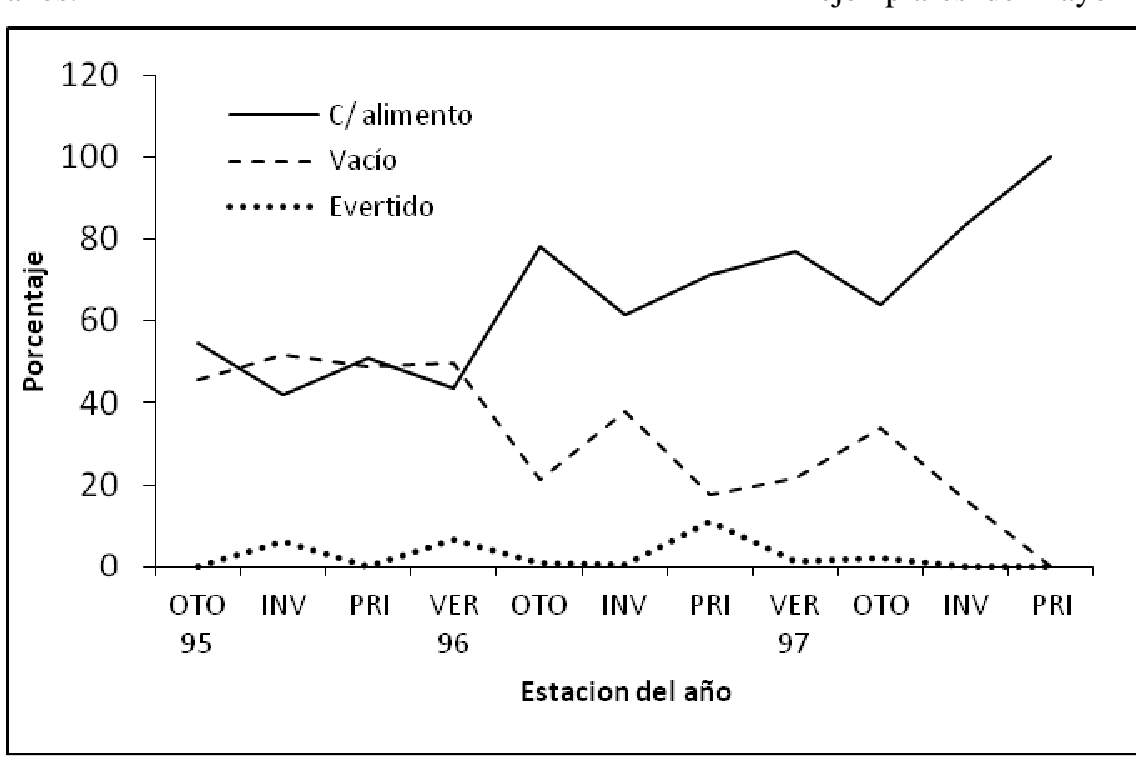

Figura 4. Porcentaje promedio de la condición de los estómagos por estación del año analizados durante 1995 - 1997.

\section{Composición de la dieta.}

La composición de la dieta de $M$. gayi para cada año y para el periodo total de estudio se muestra en la Tabla 2 donde se presentan los principales ítems alimenticios entre crustáceos, moluscos y peces, el peso de las presas identificadas en los estómagos (g), el agrupamiento de presas para el análisis dietario y el \%M para los principales grupos alimenticios de las presas identificadas. Para el periodo total las presas que forman el mayor grupo alimenticio del M. gayi son los peces teleósteos con $87 \%$ por masa de alimento ingerido, $10.4 \%$ fueron moluscos cefalópodos y sólo el $2.6 \%$ fueron crustáceos de los cuales la mayor parte constituyeron eufáusidos (Ayón \& Arones, 1997). Estos resultados son opuestos a los de Guevara (1997) quien destaca un mayor aporte en la dieta por parte de crustáceos, la reaparición de la sardina y la disminución de la anchoveta; aunque sí concuerda con el aumento del canibalismo durante el evento de El Niño.

Dentro de los peces ingeridos, la misma merluza es el principal item alimenticio (40.6\%) evidenciando un alto grado de canibalismo, siguiéndole en importancia la familia Engraulidae $(24.1 \%)$ donde predomina claramente la anchoveta y la familia Triglidae $(10.1 \%)$ con una alta predominancia del $P$. stephanophrys. Del total de las presas de peces identificados un $42,3 \%$ fueron demersales, $38,9 \%$ pelágicos y sólo el $1,3 \%$ fueron mesopelágicos. Es de destacar que para el año 1997 la variedad de la dieta se vio aumentada coincidiendo con Alamo \& Espinoza (1997) quienes determinaron que el espectro alimentario se encontró también incrementado respecto a los años 1994 y 1996, intensificándose el canibalismo por parte de ejemplares de mayor edad. Los resultados presentes concuerdan con los de Castillo et al. (1996), quien durante el otoño de 1995, encontró que la merluza se alimentó predominantemente de peces tanto para ejemplares pequeños, medianos y grandes y que también existió un fuerte canibalismo, aunque se incide en la presencia de unidades tróficas definidas en función al tamaño de los ejemplares de merluza.

Los individuos más pequeños consumen crustáceos y peces pequeños (con escasa presencia de sardina, pero sí de anchoveta), y los individuos más grandes consumen peces más grandes (falso volador y merluza) (Alamo \& Blaskovic, 1994; Alamo \& Espinoza, 1996, 1997). La Figura 5 muestra la variedad alimenticia durante todo el periodo de estudio evidenciando un gran aumento del espectro trófico en el año 1997 que podría estar relacionado con la intrusión de aguas cálidas con una mayor diversidad de la oferta alimenticia para la merluza. Guevara (1997) y Tam et al. (2006) notaron también una ampliación del espectro alimentario en el año 1997 con relación a los años 1994 y 1996, lo cual corrobora los resultados del presente estudio.

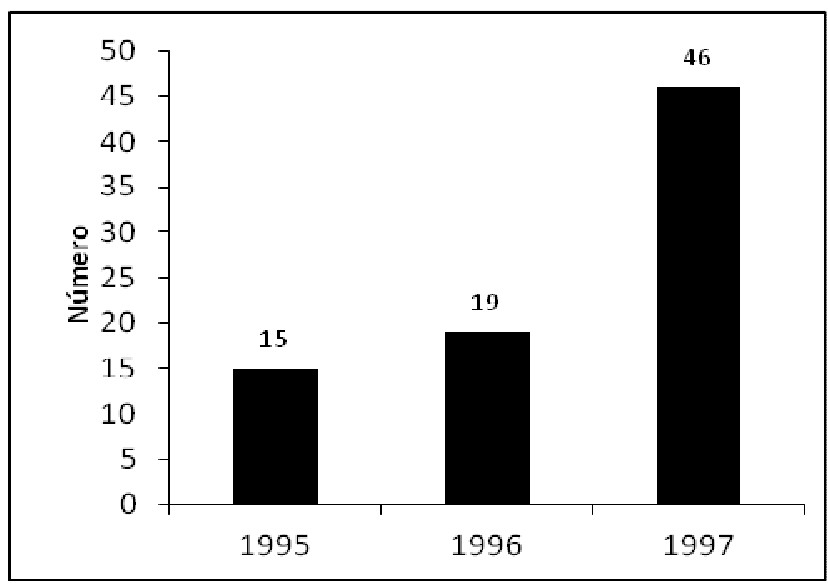

Figura 5. Número de especies registradas en la dieta alimenticia de la merluza durante el periodo de estudio (1995-1997). 
Composición de la dieta en relación a la talla.

La composición de la dieta expresada como una función de la longitud del predador (Figura 6) muestra para los tres años una preferencia alimenticia diversa, diferenciándose ésta para peces pequeños menores de $37.9 \mathrm{~cm}$ en relación con los peces más grandes mayores a $38 \mathrm{~cm}$. De esta manera, en el año 1995 para los ejemplares de merluza mayores a $38 \mathrm{~cm}$ se observa una gran predominancia de presas, como la merluza y el falso volador pero con la observación que para ejemplares de merluza mayores de $60 \mathrm{~cm}$ el
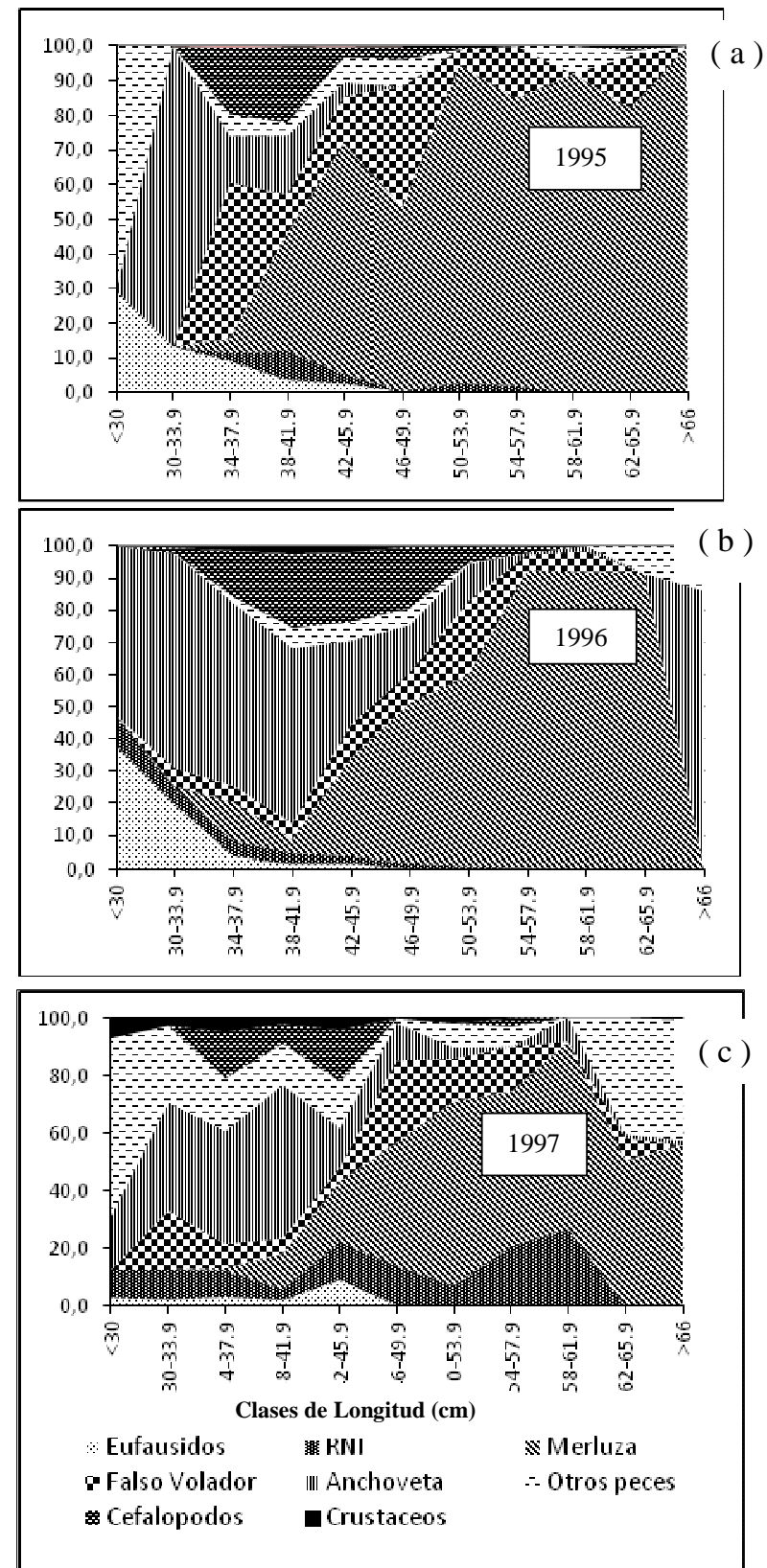

Figura 6. Composición de la dieta (\%) de las principales presas para cada grupo de longitud del M. gayi entre 1995 y 1997.

consumo se limitó exclusivamente a la propia merluza (canibalismo). Para ejemplares de merluza menores de $30 \mathrm{~cm}$ de longitud las mayores presas alimenticias están referidas a peces no identificados (RNI) y eufáusidos en mayor grado, sin embargo entre 30 y 38 $\mathrm{cm}$ se presenta una mayor variedad de presas destacando también la anchoveta y cefalópodos aunque en menor grado, estando en este último caso, constituido mayormente por el calamar Loligo sp. Para el año 1996 existe una fuerte predominancia de consumo de anchoveta y eufáusidos para los ejemplares pequeños es decir menores de $37.9 \mathrm{~cm}$; mientras que el consumo de merluza continúa siendo la principal fuente de alimentación para los ejemplares más grandes, es decir mayores a $38 \mathrm{~cm}$, siguiéndole en importancia el falso volador.

En el año 1997 se produce una modificación, ampliándose el espectro de la estructura alimenticia de la merluza en el cual los peces pequeños menores a $37.9 \mathrm{~cm}$ los principales ítems alimenticios se concentran en la anchoveta, peces diversos (caballa, jurel, anguila, bereche, anchoa, diablo, argentina, sardina y mictófidos) y crustáceos pequeños. Para los ejemplares mayores a $38 \mathrm{~cm}$ la variedad alimenticia se desagrega o diversifica en cantidad apreciándose mayor importancia en las preferencias alimenticias por la misma merluza, falso volador y en menor grado por peces no identificados $y$ otros peces diversos mencionados arriba. Los resultados presentes demuestran la importancia de la merluza mayor a los $38 \mathrm{~cm}$ de longitud cuando se evalúa el impacto de la predación sobre la misma merluza y su comportamiento altamente significativo de canibalismo en años normales (1995 y 1996) y su disminución en años en que se presenta el fenómeno de El Niño como fue en 1997. Como se puede observar existe cierta coincidencia en los resultados hallados en este trabajo con lo señalado por Guevara \& Wosnitza (2009) quienes informan que la principal dieta de la merluza para ejemplares entre 31 y $40 \mathrm{~cm}$ son los eufausidos, la anchoveta y que el canibalismo es mayor en los individuos más grandes.

Los cambios en la dieta de la merluza en relación a la talla son evidentes durante El Niño 1997, y se traduce en la similitud de la dieta que presentan las merluzas de talla pequeña y mediana. Ello permite asumir que la merluza pequeña como la mediana (30$46 \mathrm{~cm})$ es una especie oportunista ya que, contrariamente a 1995 y 1996, durante este año la contribución de peces fue mayor que la de eufáusidos.

Las diferencias interanuales encontradas en la dieta de la merluza son corroboradas por el análisis clúster aplicado a los datos totales de los tres años y que organizó a estos años en 2 grupos claramente identificados, uno que agrupa a los años 1995 y 1996 con un nivel de similaridad mayor al $60 \%$ y otro correspondiente al año 1997 claramente diferenciado con mas del $40 \%$ (Figura 7). Igualmente, tanto el análisis clúster como el escalamiento no métrico 
multidimensional agruparon a la merluza de acuerdo a los grupos de talla (Figura 8). El análisis clúster aplicado para el año 1995 organizó las 11 clases de talla en 2 grupos principales a un nivel de similaridad mayor de $40 \%$ y un grupo a un nivel de similaridad menor de 40\%. En el caso del año 1996 se observa algo muy similar aunque adicionalmente se aprecia un grupo aislado $(>66 \mathrm{~cm})$ con un nivel de similaridad bajo. En ambos casos un grupo principal estaba compuesto por merluzas pequeñas de $30-34 \mathrm{~cm}$, otro grupo por merluzas de $34-50 \mathrm{~cm}$ y un último con merluzas grandes de 50-66 cm de longitud total. Estos resultados difieren con los reportados por Tam et al. (2006) quienes realizan el agrupamiento con información de dieta colectada durante 1995 a 2002, incluyendo el año 1997/98 cuando ocurre un fuerte evento de El Niño. Analizando los grupos formados para 1997, se observan solo 2 grupos principales compuestos básicamente por merluzas de 30-46 cm y $50-66 \mathrm{~cm}$ que son muy similares a los reportados por Tam et al. (2006). Al respecto, tal como se ha demostrado en este estudio, los cambios en la dieta de la merluza debido a la ampliación del espectro alimentario y a los cambios en la abundancia de los ítems de la dieta (Ver Tabla 2, Figuras 5 y 6) dependen en gran medida de la variabilidad climática. La intrusión de aguas calientes subsuperficiales en la zona norte es variable y ello trae como consecuencia cambios en la oferta alimenticia (Espino 1990; Guevara \& Wosnitza-Mendo, 2009).

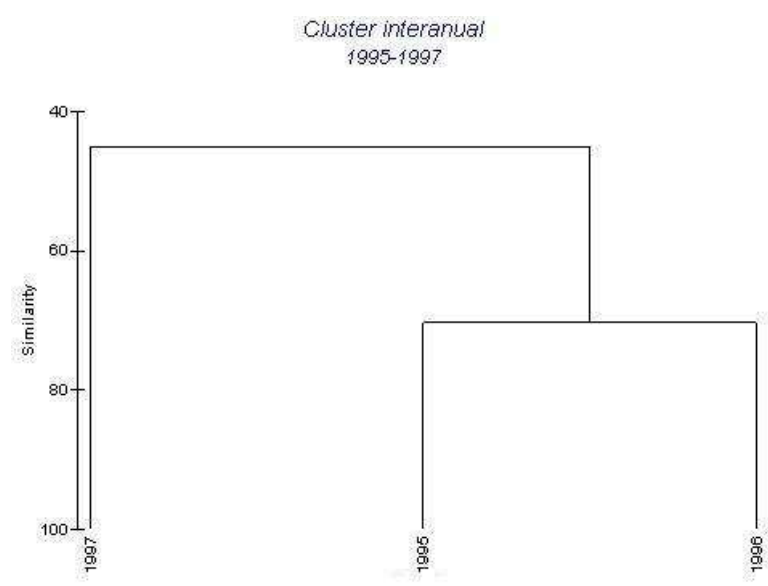

Figura 7. Análisis clúster aplicado a los datos de peso promedio por presa para los años 1995, 1996 y 1997.

$\underline{\text { Relación talla del predador -peso de la presa. }}$

La Tabla 3 muestra para los tres años de estudio la relación entre la longitud del cuerpo del predador y el peso de las presas en sus estómagos, demostrándose una relación potencial y observándose un menor valor $(b=3.701)$ en 1995 y aumentando para los siguientes años alcanzando en 1997 el mayor valor de b = 5.184 evidenciando esto una posible mayor capacidad de predación por parte del predador en relación a la presa obtenida y confirmando la alta abundancia relativa de las presas en este año. Es necesario señalar que en 1995 se presenta una gran dispersión de datos debido al bajo número de individuos muestreados razón por la

Tabla 3. Parámetros de la relación entre la longitud del cuerpo del M. gayi y el peso de los estómagos entre 1995 y 1997.

\begin{tabular}{llll}
\hline Parámetro & 1995 & 1996 & 1997 \\
\hline $\mathrm{a}$ & $2 \times 10^{-5}$ & $7 \times 10^{-7}$ & $8 \times 10^{-8}$ \\
$\mathrm{~b}$ & 3.701 & 4.674 & 5.184 \\
$\mathrm{R}^{2}$ & 0.64 & 0.93 & 0.86 \\
\hline
\end{tabular}

cual se obtiene el menor coeficiente de determinación. Variaciones estacionales de la dieta

Las variaciones estacionales de la composición alimenticia (Figura 9) muestran para las primeras clases de longitud $(34-46 \mathrm{~cm})$ un incremento regular desde otoño de 1995 hasta la primavera de 1996, pero en 1997 decrece notablemente a valores bajos en invierno de este año. Para individuos más grandes (46 - $58 \mathrm{~cm}$ ) la variación es irregular pero con incrementos en invierno para todo el periodo analizado.

Los datos del Índice de Importancia Relativa (IRI) y el índice de jerarquización (DJ) se muestran en el Anexo 1, donde se destaca la notable predominancia de los eufáusidos y la propia merluza, siguiéndoles en importancia la anchoveta, el falso volador y restos no identificados (RNI) de peces. Basado en los resultados de los análisis y específicamente para los cinco principales grupos predominantes en la dieta, los resultados en términos del IRI (Figura 10), presenta entre las especies de crustáceos más abundantes en la dieta a los eufausidos en especial la especie Euphausia mucronata (EUP) con una presencia regular y constante a lo largo de todos los periodos estacionales. Entre los teleosteos, los géneros engraulidos con la especie Engraulis ringens (ENG), los merlucidos con la especie Merluccius gayi (MER) y los Triglidae (TRI) con la especie $P$. stephanophrys representaron la dieta más importante los que fueron caracterizados por los más altos IRI.

Es importante anotar que se presentó un notable descenso de presencia de engraulidos en los estómagos de la merluza en el año 1997 (evento de El Niño) lo mismo que de eufausidos, concordante con el aumento del consumo de merluza (canibalismo) en ese mismo año y de otros peces no identificados (RNI). En este sentido, el índice de importancia relativa encontrada por Tam et al. (2006) para los crustáceos decreció con el tamaño de la merluza mientras que las presas de peces mostró una tendencia inversa, estableciendo una estrategia de alimentación marcada según el tamaño del predador, aunque este autor no realiza una evolución estacional del IRI . 

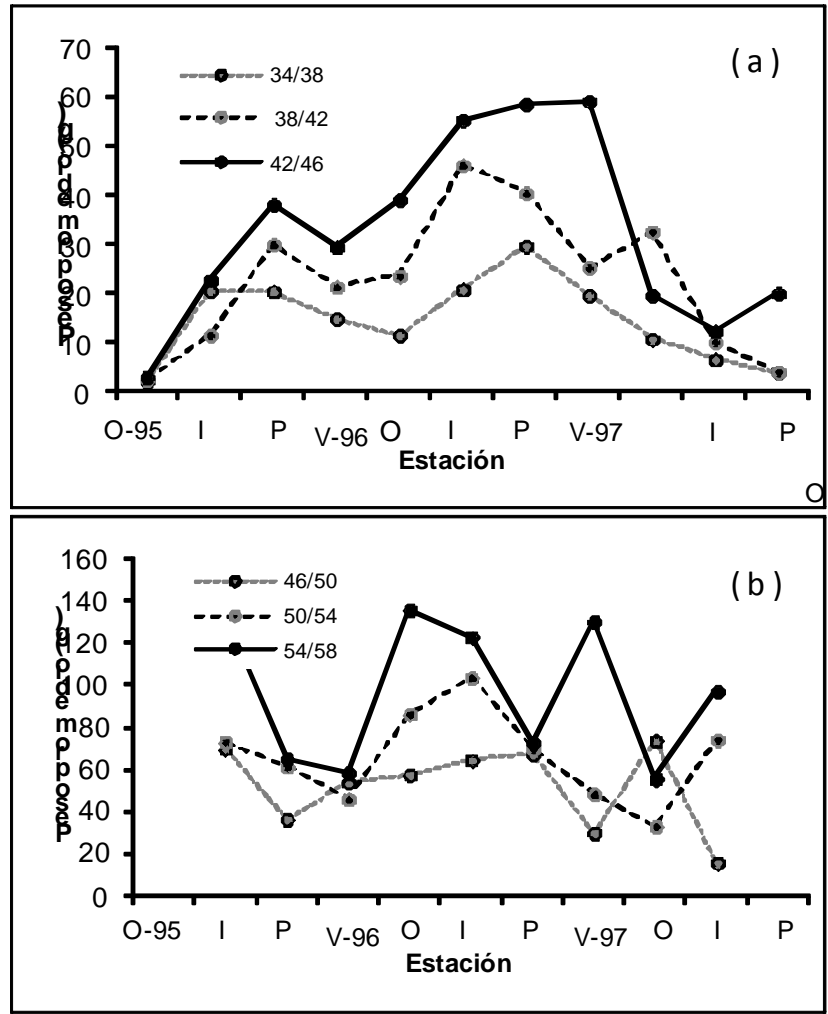

Figura 9. Variación interanual del peso (g) del contenido estomacal para los diferentes grupos de talla de la merluza frente a Paita durante 1995-1997.

Castillo et al. (1997), analizando muestras de noviembre y diciembre del año 1989 reporta que en número de individuos los grupos más importantes son los crustáceos seguidos por los teleósteos. Sus resultados muestran un alto consumo en peso de teleósteos especialmente de sardina, anchoa y anchoveta. En peso el consumo de crustáceos es menos importante especialmente la Euphausia mucronata, así el IRI para los teleósteos es aproximadamente dos veces mayor que el correspondiente a crustáceos confirmando que la merluza se alimenta principalmente de peces aunque este patrón no tiene variación estacional significativa.

El Índice de plenitud total del estómago (TFI) para el periodo total de estudio (Figura 11) muestra un comportamiento oscilante y variable pero siempre de tendencia ascendente durante los 2 primeros años (1995 y 1996), para alcanzar un máximo a fines del año 1996 reflejando buenas condiciones de ofertas alimenticias. Luego se experimenta un notorio y brusco descenso en el tercer año (1997) probablemente asociado a los cambios de las condiciones oceanográficas que se presentaron en las etapas tempranas del evento de El Niño excepcional 1997-98. Estas observaciones coinciden con lo hallado por Tam et al. (2006) que para un periodo posterior a El Niño 1997-1998 encuentra una alta variabilidad mes a mes ocasionado tal vez por una alta heterogeneidad en el suministro de alimentos como consecuencia de un evento caliente.

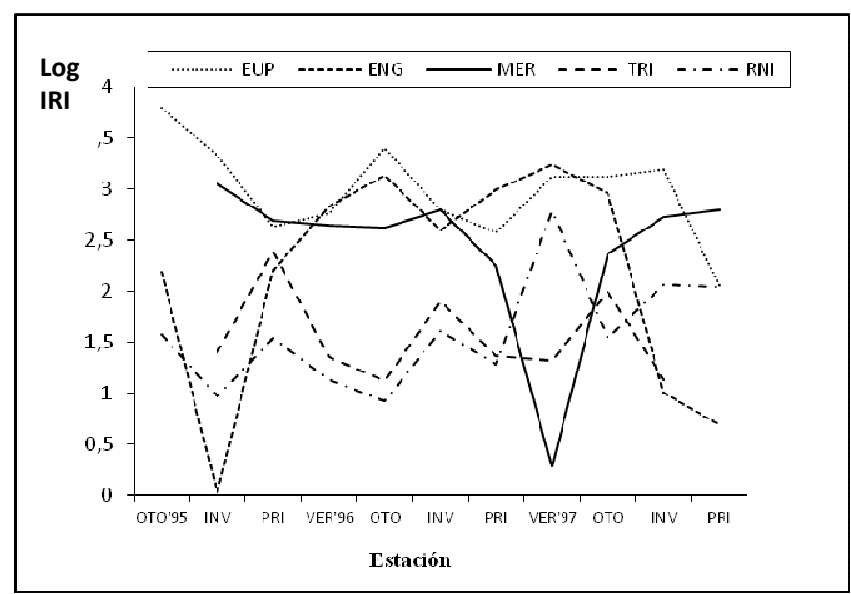

Figura 10. Evolución interanual del IRI de los principales grupos de presas de la merluza frente a Paita durante 1995-1997.

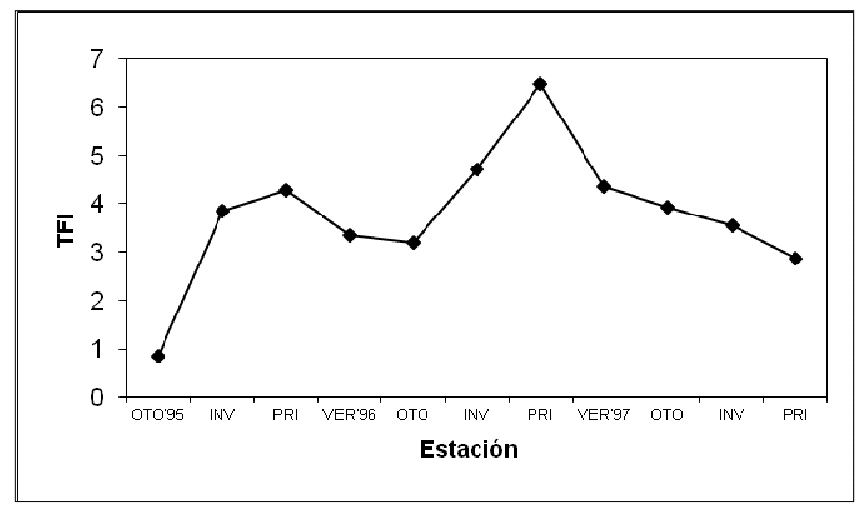

Figura 11. Variación interanual del Índice de Plenitud total del estómago (TFI) de la merluza durante 19951997.

El Índice de plenitud parcial del estómago para los grupos de presas predominantes en la dieta de la merluza (Figura 12) se observa que tanto los eufáusidos (EUP) como los peces no identificados (RNI) muestran un bajo índice aunque con un comportamiento estacional constante durante los 3 años. Sin embargo, la presa merluza Merluccius gayi (MER) presenta un ascenso continuo en este Índice, evidenciando un comportamiento particular por parte de individuos adultos predadores posiblemente ocasionado por la escasa variedad de presas disponibles hacia finales del año 1997, constituyendo por tanto el canibalismo una conducta predominante. En el caso de las especies de los géneros Engraulidae (ENG) y Triglidae (TRI) presentan índices relativamente altos constituyendo también especies importantes en la dieta de la merluza, sobretodo el falso volador aunque a fines del año 1997 presenta una notable disminución como las demás especies 
posiblemente originado por las anomalías en el mar por el calentamiento de las aguas.

De acuerdo a la evolución estacional del Índice de Intensidad de alimentación (FI) en la Figura 13, se aprecia una alta variabilidad durante todo el período de muestreo, aunque en líneas generales se presenta una ligera tendencia ascendente principalmente en el año 1997 descendiendo ligeramente hacia fines del mismo generado principalmente por el gran espectro alimentario en esta temporada (1997) apoyando la idea de que la merluza es un predador oportunista incrementando el consumo de otras presas de acuerdo a lo que ofrece el medio en esta época caracterizada por el calentamiento de las aguas por el Niño 1997-98.

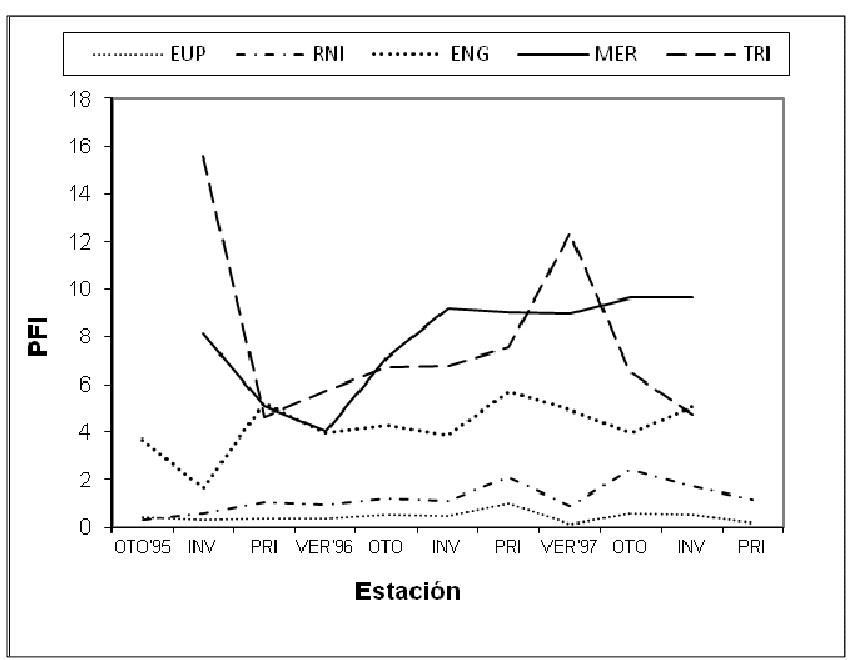

Figura 12. Variación interanual del Índice de Plenitud Parcial (PFI) para las principales presas de la merluza.

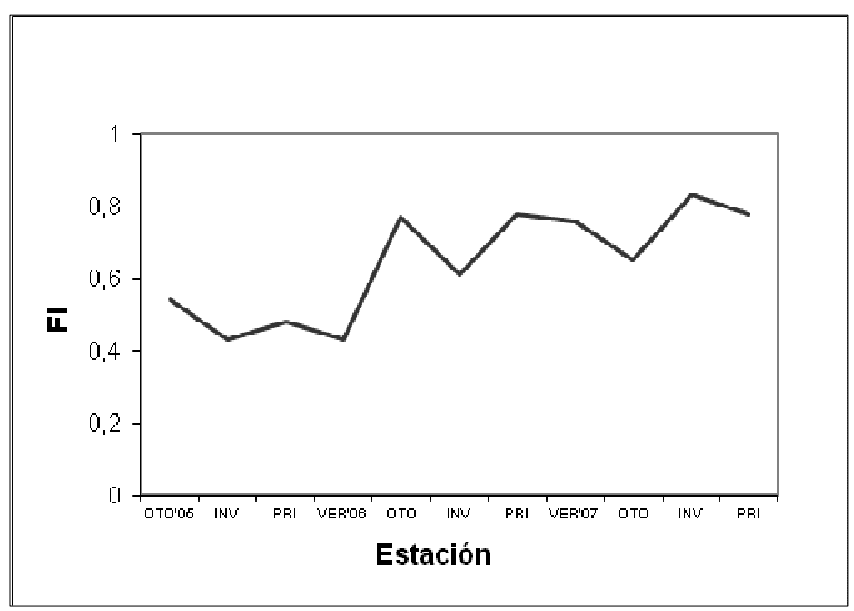

Figura 13. Variación interanual de Índice de Intensidad de Alimentación (FI) de merluza.

\section{Conclusiones.}

1. Las presas que forman el mayor grupo alimenticio del M. gayi son los peces teleósteos con $87 \%$ por masa de alimento ingerido, $10.4 \%$ fueron moluscos cefalópodos y sólo el $2.6 \%$ fueron crustáceos de los cuales la mayor parte constituyeron eufáusidos.

2. Entre los peces ingeridos, la misma merluza es el principal ítem alimenticio $(40.6 \%)$ evidenciando un alto grado de canibalismo, siguiéndole en importancia la familia Engraulidae $(24.1 \%)$ donde predomina claramente la anchoveta.

3. El año 1997, donde se presenta el evento de El Niño, se diferencia claramente en relación a los otros años y se aprecia una ampliación en el espectro trófico de la merluza, alcanzando a 46 especies con relación a las sólo 15 especies de 1995 y 19 de 1996, por lo que se considera que la hipótesis planteada es verdadera.

4. El IRI indica que los grupos predominantes en la dieta de la merluza correspondieron a la familia Eufausidae en especial la especie Euphausia mucronata, Engraulidae con la especie Engraulis ringens, Merlucidae con la especie Merluccius gayi y los Triglidae con la especie $P$. stephanophrys.

5. De acuerdo a la evolución interanual de la Plenitud total media (TFI) se presenta una tendencia ascendente hasta fines de 1996, pero a inicios del año 1997 empieza a descender notablemente.

6. El análisis cluster aplicado para los años 1995 y 1996 organizó a las clases de longitud en 3 grupos compuestos por merluzas pequeñas de $30-34 \mathrm{~cm}$, otro grupo por merluzas de $34-50 \mathrm{~cm}$ y un último con merluzas grandes de 50-66 $\mathrm{cm}$ de longitud total. Para 1997, se observan solo 2 grupos principales compuestos básicamente por merluzas de $30-46 \mathrm{~cm}$ y $50-66 \mathrm{~cm}$.

\section{Agradecimientos.}

Se agradece al Instituto Alfred Wegener (AWI) de Alemania y al Dr. Wolf Arnzt por el apoyo recibido en el financiamiento de la presente investigación como parte del proyecto: "Predation of peruvian hake and jack mackerel on the south pacific sardine off the Peruvian coast". Igualmente a la empresa "Santa Mónica" por haber facilitado la colección de muestras a bordo de sus embarcaciones.

\section{Literatura citada.}

Alamo A. \& Blaskovic V. 1994. Espectro alimentario y ración de alimentación del Merluccius gayi peruanus durante el invierno de 1994. IMARPE - DGIRH, Documento interno.

Alamo A. \& Espinoza P. 1996. Comportamiento alimenticio de la merluza durante el invierno de 1996. Crucero BIC SNP-1 9607-08. Inf. Inst. Mar Perú No 124 : 79 - 85.

Alamo A. \& Espinoza P. 1997. Espectro alimentario de la merluza durante el otoño de 1997. Crucero BIC Humboldt 9705-06. Inf. Inst. Mar Perú No 128: 47 - 55

Ayón P. \& Arones K. 1997. Comunidades de zooplancton e Ictioplancton frente a la costa norte-centro del Perú. Crucero BIC Humboldt 9705-06. Informe 128. Dic 1997. IMARPE. 
Bray J R. \& Curtis J.T. 1957. An ordination of the upland forest communities of southern Wisconsin. Ecological Monographs, 27, 325 - 349

Castillo R., Juárez L. \& Higginson L. 1989. Predación y Canibalismo en la población de la merluza peruana en el Area de Paita- Perú. Memorias del Simposio Internacional de los Recursos Vivos y las Pesquerías en el Pacífico Sudeste de 1988. Revista Pacífico Sur (Número Especial). CPPS.

Castillo R., Juárez L. \& Aldana L. 1995. Composición y consumo de alimento de la merluza peruana con especial énfasis en la ración diaria total. Inf. 112. IMARPE

Castillo R., Juárez L. \& Aldana L. 1997. Composición y consumo de alimento de la merluza peruana con especial énfasis en la ración diaria total. Inf, Prog. $N^{\circ} 71$. IMARPE.

Castillo R., Blaskovic V., Fernández F. \& Alamo A. 1996. Características biológicas de la merluza y otras especies demersales en otoño del 1995 (Crucero BIC SNP-1, 9505-06). Informe 117, Marzo 1996. Evaluación del recurso merluza. IMARPE.

Chirichigno N. 1998. Clave para identificar los peces marinos del Perú. Colaborador J. Vélez. Instituto del Mar del Perú. Publicación especial 2da ed. Revisada y actualizada.496 pp.

Clarke K. L. 1993. Non-parametric multivariate analyses of change in community structure. Austr. J. Ecol., 18, 117143.

Del Solar E. \& Alamo V. 1970. Exploración Sobre Distribución de Langostinos y Otros Crustáceos de la Zona Norte. Inform. Esp. Cruceros SNP-1 7009 (Agosto-septiembre de 1970).

Espino M. 1990. "El Niño": su impacto sobre los peces demersales del Perú. Bol. Inst. Mar Perú-Callao 14 (2). IMARPE

Espinoza P. 2000. Interacción trófica merluza-anchoveta, Existe realmente impacto por depredación?. Bol. Inst.Mar Perú, 19 (1-2), 15-20.

Espinoza P. 2001. Alimentación de la merluza peruana y sus fluctuaciones en el tiempo, p 50-54, Fórum la merluza peruana (Merluccius gayi peruanus): biología y pesquería, IMARPE, $120 \mathrm{p}$.
Guevara R. 1997. Resultados Generales del Crucero de evaluación del stock de merluza en otoño de 1997. BIC Humboldt 9705-06. Informe 128. Dic 1997. IMARPE.

Guevara R. \& Wosnitza-Mendo C. 1997. Análisis poblacional del recurso merluza (Merluccius gayi peruannus) en otoño 1997. BIC Humboldt 9705-06. Informe 128. Dic 1997. IMARPE.

Guevara R. \& Wosnitza-Mendo C. 2009. Cambios en la productividad de la merluza peruana (Merluccius gayi peruannus Ginsburg). Boletín Vol. 24 Nos 1 y 2. EneroDiciembre 2009. IMARPE.

Martori A. 1991. Alimentación de los adultos de Eoproctus asper (Dugés 1853) en la montaña media del Pirineo catalán. (España). Rev. Esp. Herp. 5:23-36.

Muck P., Espino M., Fuentes H., Wosnitza-Mendo C. \& Esquerre M. 1988. Predación de la merluza peruana (Merluccius gayi peruanus) sobre la anchoveta (Engraulis ringens), p 249 - 253. In Salzwedel and A Landa (eds.) Recursos y dinámica del ecosistema de afloramiento Peruano. Bol. Inst. Mar del Perú-Callao. Volumen Extraordinario. 382 p.

Muck P. 1989. Anchoveta consumption of Peruvian hake: a distribution and feeding model, p306-320. In D. Pauly, P. Muck, J.Mendo and I. Tsukayama (eds). The Peruvian upwelling ecosystem: dynamics and interactions. ICLARM Conference Proceedings 18, 438 p. Instituto del Mar del Perú (IMARPE) Callao, Perú.

Paz J., Casas J.M. \& Perez-Gándaras G. 1993. The feeding of cod (Gadus morhua) on Flemish Cap, 1989-90. NAFO Sci. Coun. Studies, 19: 41-50.

Pillar S. \& Wilkinson S..1995. The diet of Cape hake Merluccius capensis on the south coast of South Africa. S. Afri. J. mar. Sci. 15: $225-239$

Pinkas L., Oliphant M. \& Iverson Z.. 1971. Food habitat of albacore bluefin, tuna and bonito in California waters, California Department of Fish and Game. Fish Bulletin 152: 1-350.

Silva A. 1999. Feedings habits of John Dory, Zeus faber, off the Portuguese continental coast. J. Mar. Biol. Ass U.K. 79: $333-340$

Tam J., Purca S., Duarte L., Blaskovic V. \& Espinoza P. 2006. Changes in the diet of hake associated with El Niño 1997 - 1998 in the northern Humboldt Current ecosystem. Advances in Geosciences, 6, 63-67 
ANEXO

Tablas y figuras citadas en el texto

Tabla 2.- Porcentaje de la masa de las presas consumidas por M. gayi en la costa norte del Perú y el agrupamiento de las principales presas.

\begin{tabular}{|c|c|c|c|c|c|c|c|}
\hline \multirow{2}{*}{$\begin{array}{l}\text { Taxon } \\
\text { presa }\end{array}$} & \multirow[t]{2}{*}{ Especies } & \multirow{2}{*}{$\begin{array}{l}\text { Grupo } \\
\text { presas }\end{array}$} & \multicolumn{3}{|c|}{ Peso de las presas $(\mathrm{g})$} & \multicolumn{2}{|c|}{ TOTAL } \\
\hline & & & 1995 & 1996 & 1997 & $\mathrm{~W}(\mathrm{~g})$ & $\% \mathrm{M}$ \\
\hline Crustacea & & CRU & & & & & 1.34 \\
\hline Euphausiacea & $\begin{array}{l}\text { Euphausia m. } \\
\text { Otras sp. }\end{array}$ & EUP & 168.8 & 385.0 & $\begin{array}{r}26.5 \\
179.9\end{array}$ & $\begin{array}{l}580.4 \\
179.9\end{array}$ & 1.31 \\
\hline \multirow[t]{8}{*}{ Decapoda } & Heterocarpus sp. & & & 172.8 & 1.0 & $\begin{array}{r}173.8 \\
-5.5\end{array}$ & \\
\hline & $\begin{array}{l}\text { Pleisonika sp. } \\
\text { Pasiphaea sp. }\end{array}$ & & 3.3 & & $\begin{array}{r}24.2 \\
141.5\end{array}$ & $\begin{array}{r}27.5 \\
141.5\end{array}$ & \\
\hline & $\begin{array}{l}\text { Otras sp. (Fam. Pasip.) } \\
\text { Solenocera } s p .\end{array}$ & & 1 & 43.6 & 40.0 & 84.6 & \\
\hline & Otras sp.(Fam. Solen.) & & & 120.7 & 12.4 & 120.7 & \\
\hline & Munida sp. & & & & & 12.4 & \\
\hline & Otras sp.(Fam. Galat.) & & & & & & \\
\hline & Palinuridae & & & 25.1 & & 25.1 & \\
\hline & $\begin{array}{l}\text { Squilla p. } \\
\text { Squilla sp. }\end{array}$ & & & 1.9 & & 1.9 & \\
\hline \multirow[t]{3}{*}{ Stomatopoda } & Otras sp. & & & & 0.3 & & \\
\hline & Alona affinis & & & & 70.9 & 70.9 & \\
\hline & & & 0.6 & & 26.2 & 26.8 & \\
\hline Diplostraca & & & & & 92.0 & 92.0 & \\
\hline \multirow[t]{2}{*}{ No identifica. } & & & & & 1.9 & 1.9 & \\
\hline & & & & & 2.9 & 2.9 & \\
\hline \multicolumn{8}{|l|}{ Mollusca } \\
\hline \multirow[t]{3}{*}{ Cephalopoda } & Dosidicus gigas & DOS & 70.4 & 2156.9 & 928.3 & 3155.6 & 5.42 \\
\hline & Loligo sp. & LOL & 479.8 & 2107.3 & 275.5 & 2451.6 & 4.92 \\
\hline & Otras sp. & & & & 17.2 & 17.2 & \\
\hline \multicolumn{8}{|l|}{ Pisces } \\
\hline \multirow[t]{3}{*}{ Engraulidae } & Engraulis ringens & ENG & 473.4 & 9059.7 & 4493.2 & 14026.3 & 24.11 \\
\hline & Anchoa sp. & ANC & 19.9 & 243.7 & 436.1 & 699.8 & 1.20 \\
\hline & Otras sp. & & & 106.4 & 99.7 & 206.0 & 0.35 \\
\hline Merluccidae & Merluccius gayi & MER & 5552.0 & 11924 & 6112.9 & 23588.9 & 40.55 \\
\hline Clupeidae & Sardinops sagax & SAR & 17.4 & 386.5 & 546.5 & 950.4 & 1.63 \\
\hline \multirow[t]{2}{*}{ Triglidae } & P. stephanophrys & TRI & 1335.4 & 2699.3 & 1868.2 & 5902.9 & 10.15 \\
\hline & Otras sp. & & & & 49.5 & 49.5 & 0.09 \\
\hline Argentinidae & Argentina sp. & & 176.3 & 100.7 & 28.3 & 305.4 & 0.52 \\
\hline Moridae & Physiculus sp & & 38.9 & 120.3 & 264.1 & 423.3 & 0.73 \\
\hline Scorpaenidae & Pontinusf. & & & 72.9 & & 72.9 & 0.13 \\
\hline \multirow[t]{3}{*}{ Sciaenidae } & Stellifer minor & & 68.8 & 57.6 & & 126.4 & 0.22 \\
\hline & Larimus pacificus & & & & 85.8 & 85.8 & 0.15 \\
\hline & Otras sp. & & & & 92.4 & 92.4 & 0.16 \\
\hline \multirow[t]{3}{*}{ Myctophidae } & Lampanyctus sp. & & & & 32.5 & 32.5 & 0.06 \\
\hline & Gonichthys cocco & & & & 215.4 & 215.4 & 0.37 \\
\hline & Leuroglosus $u$. & & & & 12.9 & 12.9 & 0.02 \\
\hline Scombridae & Otras sp. & & & & 138.8 & 138.8 & 0.24 \\
\hline Carangidae & Scomber japonicus & & & & 460.0 & 460.0 & 0.79 \\
\hline Macrouridae & Trachurus murphyi & & & & 314.9 & 314.9 & 0.54 \\
\hline Ophichthyid. & Macrourus canus & & & & 15.8 & 15.8 & 0.03 \\
\hline \multirow[t]{2}{*}{ Lepidopidae } & Ophichthus p. & & & & 96.5 & 96.5 & 0.17 \\
\hline & Lepidopus sp & & & & 12.4 & 12.4 & 0.02 \\
\hline \multirow[t]{2}{*}{ No identific. } & & & & & & & \\
\hline & & RNI & 215.0 & 649.2 & 1898.3 & 2762.5 & 4.75 \\
\hline
\end{tabular}

CRU Crustacea: Incluye crustáceos excepto los Eufasidos

EUP Euphausiidae: Incluye todas las especies Euphausiidae. 


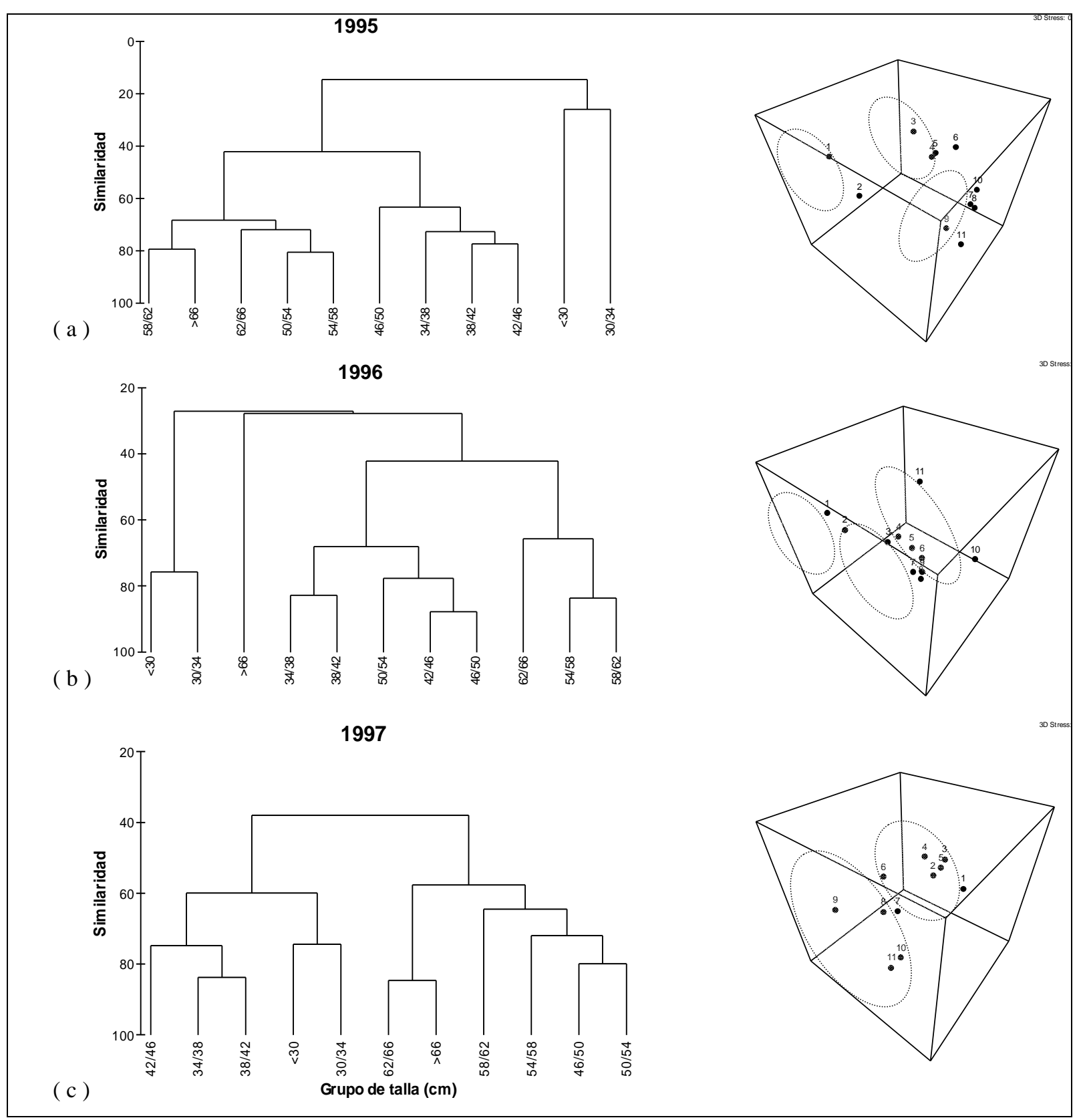

Figura 8. Dendrogramas y NMDS para los contenidos estomacales de Merluccius gayi de diferentes grupos de talla $(1=<30 \mathrm{~cm} ; 2=30 / 34 \mathrm{~cm} ; 3=34-38 \mathrm{~cm} ; 4=38-42 \mathrm{~cm} ; 5=42-46 \mathrm{~cm} ; 6=46-50 \mathrm{~cm} ; 7=50-54 \mathrm{~cm} ; 8=54-58 \mathrm{~cm}$; $9=58-62 \mathrm{~cm} ; 10=62-66 \mathrm{~cm} ; 11=>66 \mathrm{~cm} ;)$. 
Anexo 1. Índice de Importancia Relativa (Log IRI) estacional y total para cada presa y el Índice de Jerarquización (DJ)

\begin{tabular}{|c|c|c|c|c|c|c|c|c|c|c|c|c|c|}
\hline & \multicolumn{3}{|c|}{1995} & \multicolumn{4}{|c|}{1996} & \multicolumn{4}{|c|}{1997} & \multirow{2}{*}{$\begin{array}{c}\text { Log } \\
\text { IRI } \\
\text { prom } \\
\text { edio }\end{array}$} & \multirow[t]{2}{*}{ DJ } \\
\hline & OTO & INV & PRI & VER & OTO & INV & PRI & VER & OTO & INV & PRI & & \\
\hline \multicolumn{14}{|l|}{ CRUSTACEA: } \\
\hline Crustacea ind. & & & & & & & & & & -0.32 & -1.69 & -1.01 & -33.7 \\
\hline Decapoda ind. & & & & & & & & & -1.67 & -2 & & -1.84 & -61.5 \\
\hline Caridea ind. & & -1.95 & & & & & & -0.1 & -0.04 & -0.37 & 0.87 & -0.32 & -10.7 \\
\hline Macrura ind. & & & & & & & & & & & 0.61 & 0.61 & 20.4 \\
\hline Palinuridae ind. & & & & & & & & & -1.67 & & & -1.67 & -56.0 \\
\hline Galatheidae ind. & & & & & & & -1.5 & & & & & -1.50 & -50.3 \\
\hline Penaeidae ind. & & & & & -0.52 & -0.36 & -1.3 & -0.86 & & & 0.23 & -0.56 & -18.9 \\
\hline Solenocera sp. & & & & & & 1.36 & & & & & & 1.36 & 45.6 \\
\hline Heterocarpus sp. & & & & & & 1.46 & & & & & 0.55 & 1.01 & 33.7 \\
\hline Plesionika trispinus & 0.63 & -1.53 & & & & & & & & -1.22 & 2.11 & 0.00 & -0.1 \\
\hline Pasiphaea sp. & & & & & & & & -0.51 & 1.05 & 2.4 & 1.94 & 1.22 & 40.9 \\
\hline P. monodon & & & & & & & & & & & -1 & -1.00 & -33.5 \\
\hline Munida sp. & & & & & -1.05 & & & & & & & -1.05 & -35.2 \\
\hline Euphausiacea ind. & 3.8 & 3.33 & 2.64 & 2.76 & 3.4 & 2.8 & 2.58 & 3.13 & 3.13 & 3.2 & 2.05 & 2.98 & 100.0 \\
\hline Stomatopoda ind. & & & & & & & & 1.04 & -1.67 & -0.58 & 0.33 & -0.22 & -7.4 \\
\hline Squilla panamensis & & & & & & & & & & -1.04 & -1.52 & -1.28 & -42.9 \\
\hline Squilla $s p$. & -0.28 & & & & & & & -1.61 & 0.15 & 0.38 & 0.43 & -0.19 & -6.2 \\
\hline Alona affinis & & & & & & & & & & -0.18 & & -0.18 & -6.0 \\
\hline Megalopa & & & & & & & & -0.38 & -1.67 & & & -1.03 & -34.4 \\
\hline \multicolumn{14}{|l|}{ TELEOSTOMI } \\
\hline RNI & 1.58 & 0.97 & 1.54 & 1.14 & 0.93 & 1.61 & 1.28 & 2.79 & 1.54 & 2.06 & 2.04 & 1.59 & 53.3 \\
\hline Triglidae & & & & & & & & & -1.67 & -0.62 & & -1.15 & -38.4 \\
\hline P. stephanophrys & & 1.4 & 2.39 & 1.36 & 1.12 & 1.9 & 1.37 & 1.32 & 1.99 & 1.13 & & 1.55 & 52.1 \\
\hline Engraulidae & & & & & & & 0.39 & -0.92 & -1.63 & 0.53 & 1.3 & -0.07 & -2.2 \\
\hline Engraulis ringens & 2.19 & 0.03 & 2.2 & 2.83 & 3.14 & 2.6 & 3 & 3.24 & 2.96 & 1.01 & 0.69 & 2.17 & 72.8 \\
\hline Anchoa sp. & 1.16 & & & -0.22 & -0.48 & -0.09 & -0.56 & & 0.88 & 1.21 & 1.73 & 0.45 & 15.2 \\
\hline Anchovia rastralis & & & & & & & & & & -0.16 & & -0.16 & -5.4 \\
\hline Sciaenidae ind. & & & & & & & & -0.06 & & -0.72 & 1.82 & 0.35 & 11.6 \\
\hline Larimus pacificus & & & & & & & & & & & 0.32 & 0.32 & 10.7 \\
\hline Stellifer minor & 0.79 & & 0.53 & & & -0.67 & & & & & & 0.22 & 7.3 \\
\hline Larimus sp. & & & & & & & & & & & 0.6 & 0.60 & 20.1 \\
\hline Myctophidae ind. & & & & & & & & -1.31 & & 2.08 & 0.85 & 0.54 & 18.1 \\
\hline Gonichthys cocco & & & & & & & & & & 1.32 & & 1.32 & 44.2 \\
\hline Lampanyctus sp. & & & & & & & & & & -0.29 & & -0.29 & -9.7 \\
\hline M. gayi peruanus & & 3.06 & 2.69 & 2.65 & 2.62 & 2.8 & 2.25 & 0.29 & 2.37 & 2.73 & 2.8 & 2.43 & 81.3 \\
\hline S. sagax sagax & & & -0.25 & 0.41 & -0.004 & -0.95 & 0.62 & -0.14 & 0.89 & & & 0.08 & 2.8 \\
\hline T. symmetricus & & & & & & & & & & 0.65 & -0.3 & 0.18 & 5.9 \\
\hline Argentina aliceae & & 0.67 & & & & 0.23 & & & & & 0.83 & 0.58 & 19.3 \\
\hline Seriolella violacea & & & & & & & & & & & 0.75 & 0.75 & 25.1 \\
\hline Ophichthus pacifici & & & & & & & & & -1.67 & & -0.02 & -0.85 & -28.3 \\
\hline S. japonicus & & & & & & & & & & 0.34 & & 0.34 & 11.4 \\
\hline Moridae & & & & & & & & -0.85 & & 0.89 & & 0.02 & 0.7 \\
\hline Physiculus talarae & & -0.28 & & 0.1 & & & & & & -1.04 & 0.97 & -0.06 & -2.1 \\
\hline Lepidopus sp. & & & & & & & & & & -1.22 & & -1.22 & -40.9 \\
\hline Scorpaena sp. & & & & & & -0.56 & & & & & & -0.56 & -18.8 \\
\hline Pontinus furcirhinus & & & & & & & & & & & 0.4 & 0.40 & 13.4 \\
\hline Vinciguerria sp. & & & & & & & & & & & -0.82 & -0.82 & -27.5 \\
\hline L. urotranus & & & & & & & & & & -1.5 & & -1.50 & -50.3 \\
\hline Macrourus canus & & & & & & & & & & -1.09 & & -1.09 & -36.5 \\
\hline \multicolumn{14}{|l|}{ MOLLUSCA } \\
\hline Cephalopoda ind. & & & & & & & & & & -1.04 & & -1.04 & -34.9 \\
\hline Dosidicus gigas & & -0.88 & 0.46 & 0.34 & & 0.34 & 2.27 & 1.95 & & -0.45 & -0.63 & 0.43 & 14.2 \\
\hline Loligo gahi & & & 2.08 & 0.39 & -1.29 & 0.65 & 2.37 & 0.91 & -0.61 & -0.1 & -0.85 & 0.39 & 13.2 \\
\hline Abraliopsis affinis & & & & & & & & & & -0.18 & & -0.18 & -6.0 \\
\hline
\end{tabular}


1 Facultad de Pesquería, Universidad Nacional Agraria La Molina, Apdo. 12-056, Lima. 100-Perú, horrego@lamolina.edu.pe.

2 Facultad de Pesquería, Universidad Nacional Agraria La Molina, Apdo. 12-056, Lima. 100-Perú, jmendo@lamolina.edu.pe. 